\title{
French experience of balloon pulmonary angioplasty for chronic thromboembolic pulmonary hypertension
}

\author{
Philippe Brenot ${ }^{1,2,3,6}$, Xavier Jaïs ${ }^{1,3,4,6}$, Yu Taniguchi ${ }^{1,3,4}$, \\ Carlos Garcia Alonso ${ }^{1,2,3}$, Benoit Gerardin ${ }^{1,2,3}$, Sacha Mussot ${ }^{1,3,5}$, \\ Olaf Mercier ${ }^{1,3,5}$, Dominique Fabre ${ }^{1,3,5}$, Florence Parent ${ }^{1,3,4}$, Mitja Jevnikar ${ }^{1,3,4}$, \\ David Montani (1) ${ }^{1,3,4}$, Laurent Savale (10 ${ }^{1,3,4}$, Olivier Sitbon (10 ${ }^{1,3,4}$, Elie Fadel ${ }^{1,3,5^{\prime}}$ \\ Marc Humbert (10) $1,3,4$ and Gérald Simonneau ${ }^{1,3,4}$
}

\begin{abstract}
Affiliations: 'Univ. Paris-Sud, Faculté de Médecine, Université Paris-Saclay, Le Kremlin-Bicêtre, France. ${ }^{2}$ Service de Radiologie, Hôpital Marie Lannelongue, Le Plessis Robinson, France. ${ }^{3}$ Inserm UMR_S 999 , Hôpital Marie Lannelongue, Le Plessis Robinson, France. ${ }^{4}$ AP-HP, Service de Pneumologie, Centre de Référence de l'Hypertension Pulmonaire, Hôpital Bicêtre, Le Kremlin-Bicêtre, France. ${ }^{5}$ Service de Chirurgie Thoracique, Hôpital Marie Lannelongue, Le Plessis Robinson, France. ${ }^{6}$ Both authors contributed equally to the study.
\end{abstract}

Correspondence: Xavier Jaïs, Service de Pneumologie, Hôpital Bicêtre, 78 rue du Général Leclerc, 94275 Le Kremlin-Bicêtre Cedex, France. E-mail: xavier.jaislagmail.com

@ERSpublications

Refined balloon pulmonary angioplasty improves short-term symptoms, oxygenation, exercise capacity and haemodynamics in inoperable CTEPH patients with an acceptable risk-benefit ratio http://bit.ly/ $2 \mathrm{UjaHhb}$

Cite this article as: Brenot $\mathrm{P}$, Jaïs $\mathrm{X}$, Taniguchi $\mathrm{Y}$, et al. French experience of balloon pulmonary angioplasty for chronic thromboembolic pulmonary hypertension. Eur Respir J 2019; 53: 1802095 [https:// doi.org/10.1183/13993003.02095-2018].

\section{ABSTRACT}

Aims: To evaluate safety and efficacy of balloon pulmonary angioplasty (BPA) in a large cohort of patients with chronic thromboembolic pulmonary hypertension (CTEPH).

Methods: From 2014 to 2017, 184 inoperable CTEPH patients underwent 1006 BPA sessions. Safety and efficacy during the first 21 months (initial period) were compared with those of the last 21 months (recent period). A total of 154 patients had a full evaluation after a median duration of 6.1 months.

Results: Overall, there was a significant improvement in New York Heart Association functional class, 6-min walk distance (mean change $+45 \mathrm{~m}$ ), and a significant decrease in mean pulmonary artery pressure (PAP) and in pulmonary vascular resistance (PVR) by $26 \%$ and $43 \%$, respectively. The percentage decreases of mean PAP and PVR were $22 \%$ and $37 \%$ in the initial period versus $30 \%$ and $49 \%$ in the recent period, respectively $(\mathrm{p}<0.05)$. The main complications included lung injury, which occurred in $9.1 \%$ of 1006 sessions ( $13.3 \%$ in the initial period versus $5.9 \%$ in the recent period; $\mathrm{p}<0.001$ ). Per-patient multivariate analysis revealed that baseline mean PAP and the period during which BPA procedure was performed (recent versus initial period) were the strongest factors related to the occurrence of lung injury. 3-year survival was $95.1 \%$.

Conclusion: This study confirms that a refined BPA strategy improves short-term symptoms, exercise capacity and haemodynamics in inoperable CTEPH patients with an acceptable risk-benefit ratio. Safety and efficacy improve over time, underscoring the unavoidable learning curve for this procedure. 


\section{Introduction}

Chronic thromboembolic pulmonary hypertension (CTEPH) is caused by the obstruction of the pulmonary arteries with non-resolving, organised fibrotic clots leading to elevated pulmonary vascular resistance (PVR), severe pulmonary hypertension (PH), right heart failure and, ultimately, death [1-5].

Pulmonary endarterectomy (PEA) remains the recommended treatment for patients with operable CTEPH [6-12]. However, about $40 \%$ of CTEPH patients are ineligible for surgery due to distal lesions or the presence of comorbidities [10]. Today, balloon pulmonary angioplasty (BPA), an endovascular procedure to widen narrowed -or obstructed pulmonary arteries, is an emerging treatment option for patients with inoperable CTEPH [12, 13]. This technique was first developed for treating congenital stenosis of pulmonary arteries [14]. In CTEPH, FEINSTEIN et al. [15] reported, in 2001, a first case series of 18 patients with a modest efficacy on pulmonary haemodynamics and a high rate of severe, potentially lethal complications.

Over more recent years, with refinements to the technique, several limited cases series, mainly from Japan, have reported major improvements in the safety and efficacy of BPA [16-24]. These encouraging results have been recently confirmed in a multicentre registry of 308 patients with CTEPH treated with BPA in seven centres in Japan between 2004 and 2013. This study demonstrated a favourable effect of BPA on haemodynamics with a decrease in PVR of more than 50\%. However, the complication rate remained elevated and mainly included non-severe lung injury which occurred in $17.8 \%$ of cases [25].

In Europe, over recent years, the number of centres initiating a BPA programme is growing rapidly. A first series of $56 \mathrm{CTEPH}$ patients, who underwent BPA in Germany, has been recently reported and demonstrated a significant haemodynamic improvement associated with a mortality rate of $1.8 \%$ [26].

We report the experience of BPA at the French Reference Centre for Pulmonary Hypertension (Paris-Sud University, Hospital Bicetre and Hospital Marie Lannelongue), where a BPA programme was initiated in 2014 .

\section{Methods}

\section{Patient selection}

All patients referred to Paris-Sud University for suspicion of CTEPH were evaluated during a multidisciplinary meeting including experienced surgeons for PEA, interventional radiologists/ cardiologists, radiologists experienced in pulmonary vascular imaging and pulmonologists with expertise in $\mathrm{PH}$, as recommended by current guidelines [12]. Patients underwent a complete workup, including medical history and comorbidity assessment, ventilation/perfusion lung scan, spiral computed tomography (CT) scan with mandatory bi-planar reconstructions, digital subtraction pulmonary angiography and right heart catheterisation. Eligibility for BPA was decided on the basis of a consensus among the multidisciplinary team. All the patients were informed about the potential risks and benefits of this interventional procedure and provided written informed consent.

\section{Patient evaluation before and after BPA}

All patients underwent a comprehensive clinical evaluation before the first BPA (baseline), before each BPA session and 3-6 months after the last BPA. Assessment at baseline and at the last evaluation included New York Heart Association (NYHA) functional class, 6-min walk distance (6MWD), blood gases on room air, serum levels of N-terminal pro-brain natriuretic peptide (NT-proBNP) and complete right heart catheterisation. Assessment before each new BPA session included NYHA functional class, laboratory studies and measurement of pulmonary artery pressure.

\section{BPA technique}

Four experienced operators (two interventional cardiologists and two interventional radiologists) performed the BPA procedures. All operators had more than 10 years of experience practising endovascular treatment. BPA was performed using techniques similar to those previously described [16, 18]. We approached the pulmonary arteries through the right femoral vein using peripheral guiding sheath (6 French Destination $65 \mathrm{~cm}$; Terumo, Tokyo, Japan; 7 French ArrowFlex $80 \mathrm{~cm}$; Teleflex, Durham, NC, USA), with anticoagulation continued with a dosage of vitamin $\mathrm{K}$ antagonist to maintain an INR 3.0. A right heart catheterisation was performed at the beginning of the procedure to measure mean pulmonary artery pressure (mPAP) and cardiac output by thermodilution. A 6 French guide catheter (Launcher, Multipurpose, Judkins right and left 4.0, Amplatz right and left; Medtronic, MN, USA) was inserted through the peripheral guiding sheath and was advanced to the target vessels. A dose of heparin (20003000 units) was then administered. Based on selective pulmonary angiography, a 0.014-inch guide wire (Whisper MS or Pilot 50-150; Abbott Vascular, Santa Clara, CA, USA; PT2; Boston Scientific, Marlborough, MA, USA) was passed across the target lesion. While selecting the target vessels, the lower 
lobe lesions were preferentially dilated because the pulmonary blood flow at this site is relatively high, thus lowering mPAP. Ring-like stenosis, web and subtotal lesions were targeted in priority. Tortuous lesions were exceptionally targeted and total occlusion lesions were rarely targeted. To approach total occlusion lesions, balloon support or medium tip weight wires (tip load 2.5-2.9 g) were used. Furthermore, in cases of severe stenosis, abrupt narrowing or complete obstruction, a $2.0-\mathrm{mm}$ balloon catheter was initially used to dilate the lesions. Subsequently, the lesions were dilated to an appropriate size using $2.0-\mathrm{mm}$ to $9.0-\mathrm{mm}$ balloon catheters depending on vessel diameter (NC TREK or Viatrac 14 Plus; Abbott Vascular, Santa Clara, CA, USA; Ryujin Terumo, Tokyo, Japan). We treated two to 10 segmental or subsegmental arteries in each procedure session according to patient severity, time of procedure $(<2 \mathrm{~h})$ and amount of contrast media given. Two BPA sessions were performed at 2- or 3-day intervals during one hospital admission. Catheterisation was repeated at an interval of 3-4 weeks and additional BPA sessions were performed until mPAP below $30 \mathrm{mmHg}$ was achieved, and/or when we considered that we have treated all accessible lesions.

\section{Definition of complications related to BPA}

During the 6th World Symposium on Pulmonary Hypertension, the task force on CTEPH proposed a definition and classification of complications related to BPA procedures which have been recently published in the European Respiratory Journal. In that publication, it was stated lung injury appears to be the most common complication and is characterised by lung opacities on chest radiograph or CT scan, with or without hypoxaemia, and may or may not be associated with haemoptysis [27]. The cause of lung injury has been thought to be reperfusion oedema, as is the case for PEA, however recent insight has shown that the main underlying cause was haemorrhage complication due to distal vascular injury which was provoked by iatrogenic wire injury or over dilation of the lesion [13, 21, 27-29].

In our study, complications related to BPA were defined as follows: 1) lung injury (presence of lung opacities on chest radiograph and/or CT scan with or without hypoxaemia and with or without haemoptysis; the severity of lung injury was considered as mild (no treatment), moderate (requiring supplemental oxygenation) or severe (requiring mechanical ventilation and/or extracorporeal membrane oxygenation)); 2) haemoptysis; 3) pulmonary artery perforation; 4) pulmonary artery dissection; and 5) renal dysfunction.

We analysed the safety and efficacy of BPA for the overall population. We also compared the safety and efficacy results of BPA during the initial period of the programme, defined by patients who underwent BPA during the first 21 months (between February 2014 and October 2015) versus the recent period, defined by the last 21 months (between November 2015 and July 2017).

\section{Statistical analysis}

Data were stored in a personal computer-based data spreadsheet. All statistical analyses were performed using GraphPad Prism version 5 (GraphPad Software, La Jolla, CA, USA) and SPSS Statistics 17.0 (IBM, Armonk, NY, USA). Continuous variables are expressed as mean \pm SD or median and interquartile range, according to variable distribution. Differences in continuous variables, such as patients' age, 6MWD and haemodynamic characteristics, were compared using the independent t-test for normally distributed variables and the Mann-Whitney U-test for non-normally distributed variables. Categorical variables, such as gender, NYHA functional class and use of pulmonary arterial hypertension (PAH) targeted therapy or incidence of complications were expressed as number and percentage and were compared using the chi-square test for independence or Fisher's exact test. Per-patient logistic regression analysis was used to evaluate the predictive variables for lung injury. Variables with a p-value $<0.2$ were included in the multivariate analysis.

The Kaplan-Meier method was used to estimate overall survival. For the survival analysis, the date of first BPA session was used as the start point to determine length of survival. The cut-off date was December 31, 2017. Patients who underwent lung transplantation were censored at the date of transplantation.

For all analyses, the level of statistical significance was set at $\mathrm{p}<0.05$.

\section{Results}

Patient characteristics and BPA procedures

Between February 2014 and July 2017 (42 months), 212 patients were considered as eligible for BPA. 28 patients were excluded from the overall analysis because included in the ongoing randomised controlled trial comparing balloon pulmonaRy angioplasty versus riociguAt for the treatment of non-operable Chronic thromboembolic pulmonary hypertEnsion (RACE study, NCT 02634203). Thus, 184 patients were analysed for safety. As second right heart catheterisation after the final BPA had not yet been performed in 30 of the patients at the time of cut-off date, the efficacy analysis was evaluated in 154 patients (figure 1). 


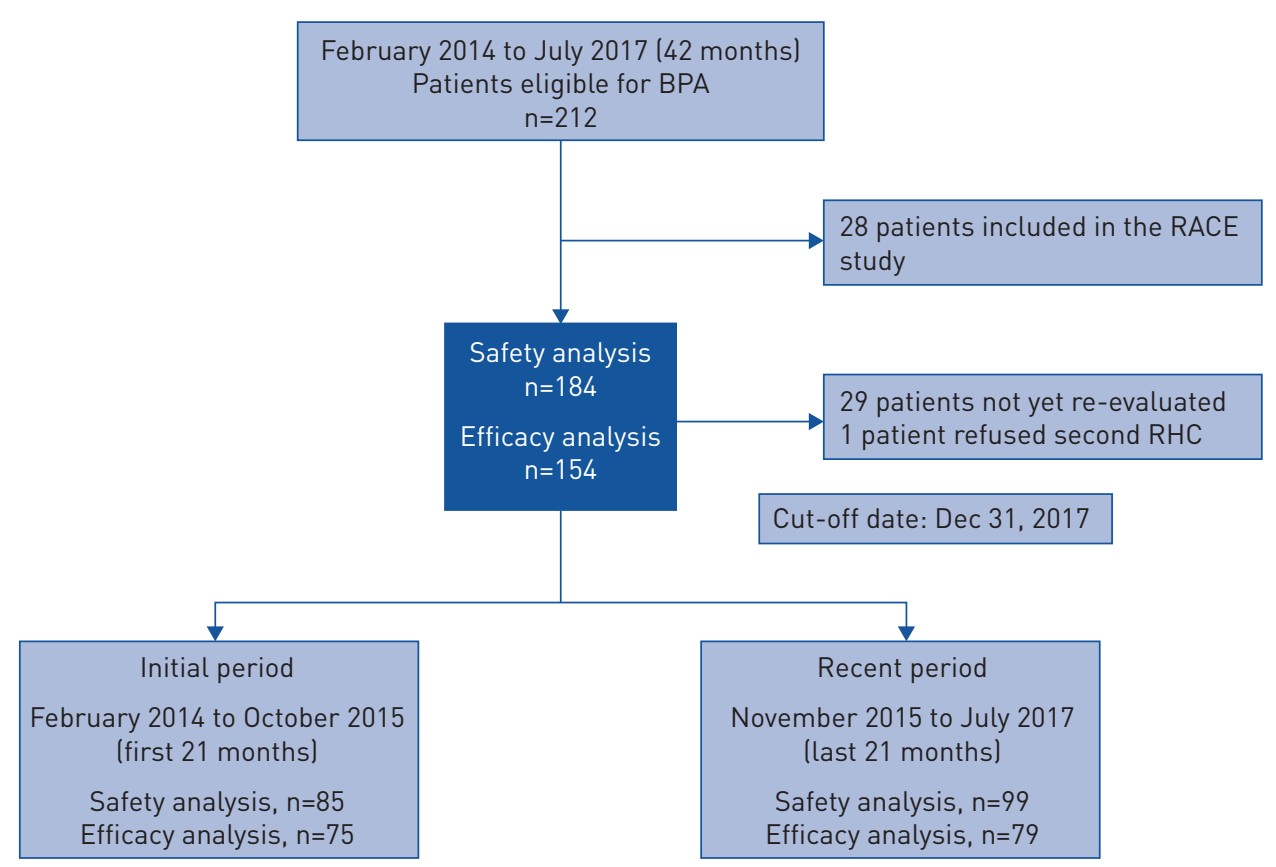

FIGURE 1 Flow chart of the study population. BPA: balloon pulmonary angioplasty; RHC: right heart catheterisation.

Baseline characteristics of the overall population $(n=184)$ are depicted in table 1.

The mean age of the patients was $63 \pm 14$ years and $51 \%$ were male. A history of acute venous thromboembolism was reported in 135 patients (73\%). At the time of BPA, 50 patients (27\%) had medical conditions increasing the risk of CTEPH, including cardiac pacemaker or indwelling catheter $(n=22)$, splenectomy $(\mathrm{n}=18)$, antiphospholipid syndrome $(\mathrm{n}=7)$ and myeloproliferative disorder $(\mathrm{n}=3)$. Pre-BPA, $94 \%$ of patients were in NYHA functional class II or III and $62 \%$ had received at least one PAH-targeted therapy for at least 3 months.

The main indications for BPA were clot inaccessibility to PEA (81\%) and persistent or recurrent PH after surgery $(8 \%)$. For patients who had been previously operated on, the period from PEA to BPA was $68 \pm 51$ months. During the study period, the 184 patients underwent a total of 1006 sessions with a mean of 5.2 \pm 2.4 sessions per patient during the initial period and $5.7 \pm 2.1$ during the recent period (no significant difference). The median (range) number of treated segments in each patient was 14.0 (10.0-20.0) during the initial period and 16.0 (12.0-21.0) during the recent period $(\mathrm{p}=0.076)$.

The fluoroscopy time was $44.4 \pm 14.9 \mathrm{~min}$ per procedure in the initial period and $42.1 \pm 12.3$ in the recent period, respectively $(\mathrm{p}=0.023)$. The median (interquartile range) duration from first BPA to re-evaluation was $6.1(4.5-7.5)$ months.

\section{Effects of BPA}

In the 154 patients who underwent a complete clinical and haemodynamic evaluation after the final BPA procedure, a significant improvement in NYHA functional class, 6MWD and arterial partial pressure of oxygen $\left(\mathrm{PaO}_{2}\right)$ was observed. Haemodynamic parameters, including right atrial pressure, mPAP, cardiac index and PVR were also significantly improved. The percentage decreases in mPAP and PVR were $22 \%$ and $37 \%$ in the initial period compared to $30 \%$ and $49 \%$ in the recent period, respectively. This greater improvement in mPAP and PVR during the recent period was statistically significant ( $\mathrm{p}=0.017$ and 0.006 , respectively). Table 2 shows the effects of BPA on symptoms, exercise capacity and haemodynamics in the initial and recent periods.

\section{Complications of BPA}

Complications related to BPA occurred in 113 of 1006 sessions $(11.2 \%$ of all sessions and $46 \%$ of all patients) and included lung injury (9.1\% of all sessions and $34 \%$ of all patients), haemoptysis $(7.1 \%$ of all sessions), pulmonary artery perforation (2.8\% of all sessions), pulmonary artery dissection ( $1.9 \%$ of all sessions) and renal dysfunction ( $0.2 \%$ of all sessions). The vast majority of lung injury occurred during the first $24 \mathrm{~h}$. Only four patients developed documented lung injury after $24 \mathrm{~h}$. Complications per session are summarised in table 3 . The rate of complications was reduced from $15.8 \%$ of sessions in the initial 


\begin{tabular}{|c|c|}
\hline \multicolumn{2}{|l|}{ Characteristics } \\
\hline Age years & $63 \pm 14$ \\
\hline Males & $94(51)$ \\
\hline NYHA FC \% (I/II/III/IV) & $1.1 / 35.0 / 59.0 / 4.9$ \\
\hline 6MWD m & $397 \pm 117$ \\
\hline History of VTE & 135 (73) \\
\hline \multicolumn{2}{|l|}{ Associated medical conditions } \\
\hline Splenectomy & $18(9.8)$ \\
\hline Pacemaker/indwelling catheter & $22(12.0)$ \\
\hline Myeloproliferative disorder & $3(1.6)$ \\
\hline APS & $7(3.8)$ \\
\hline \multicolumn{2}{|l|}{ Lung function tests } \\
\hline TLC \% pred & $90.7 \pm 15.2$ \\
\hline FVC \% pred & $99.7 \pm 21.9$ \\
\hline FEV $1 \%$ pred & $89.1 \pm 20.1$ \\
\hline Dıco \% pred & $61.2 \pm 13.6$ \\
\hline \multicolumn{2}{|l|}{ Haemodynamics } \\
\hline Systolic PAP mmHg & $75.6 \pm 17.5$ \\
\hline Diastolic PAP mmHg & $24.5 \pm 7.4$ \\
\hline Mean PAP mmHg & $44.1 \pm 9.8$ \\
\hline Mean RAP mmHg & $8.3 \pm 4.0$ \\
\hline PAWP $\mathrm{mmHg}$ & $9.6 \pm 3.5$ \\
\hline Cardiac output L.min ${ }^{-1}$ & $4.89 \pm 1.30$ \\
\hline Cardiac index $\mathrm{L} \cdot \mathrm{min}^{-1} \cdot \mathrm{m}^{-2}$ & $2.71 \pm 0.64$ \\
\hline PVR dyn $\cdot \mathrm{s} \cdot \mathrm{cm}^{-5}$ & $610 \pm 255$ \\
\hline $\mathrm{SvO}_{2} \%$ & $62.2 \pm 7.2$ \\
\hline \multicolumn{2}{|l|}{ PAH therapy } \\
\hline sGC stimulator & $59(32.1)$ \\
\hline ERA & $73(39.7)$ \\
\hline PDE5-I & $47(25.5)$ \\
\hline Prostacyclin analogue & $13(7.1)$ \\
\hline Medications \% (none/single/double/triple) & $38.0 / 26.6 / 28.3 / 7.1$ \\
\hline \multicolumn{2}{|l|}{ Indication for BPA } \\
\hline Clot inaccessibility & $149(81.0)$ \\
\hline Unfavourable risk-benefit ratio for PEA & $16(8.7)$ \\
\hline Refusal of PEA & $4(2.2)$ \\
\hline Post-PEA & $15(8.2)$ \\
\hline \multicolumn{2}{|c|}{$\begin{array}{l}\mathrm{n}=184 \text {. Data are presented as mean } \pm \text { SD or } \mathrm{n}(\%) \text {, unless otherwise stated. NYHA FC: New York Heart } \\
\text { Association functional class; 6MWD: 6-min walk distance; VTE: venous thromboembolism; APS: } \\
\text { antiphospholipid syndrome; TLC: total lung capacity; FVC: forced vital capacity; FEV1: forced expiratory } \\
\text { volume in } 1 \mathrm{~s} \text {; DLCO: diffusing capacity for carbon monoxide; PAP: pulmonary artery pressure; RAP: right } \\
\text { atrial pressure; PAWP: pulmonary artery wedge pressure; PVR: pulmonary vascular resistance; } \mathrm{SvO}_{2} \text { : mixed } \\
\text { venous oxygen saturation; PAH: pulmonary arterial hypertension; sGC: soluble guanylate cyclase; ERA: } \\
\text { endothelin receptor antagonist; PDE5-I: phosphodiesterase-5 inhibitor; BPA: balloon pulmonary } \\
\text { angioplasty; PEA: pulmonary endarterectomy. }\end{array}$} \\
\hline
\end{tabular}

period to $7.7 \%$ in the recent period, respectively $(\mathrm{p}<0.001)$. Similarly, the percentage of severe lung injury decreased from $10.4 \%$ of sessions during the initial period to $1.8 \%$ during the recent period, respectively $(\mathrm{p}<0.001)$. Severe complications requiring noninvasive positive pressure ventilation were observed in $34.1 \%$ of patients and $9 \%$ of sessions during the initial period and in $5.1 \%$ of patients and $1.6 \%$ of sessions during the recent period, respectively $(\mathrm{p}<0.001)$. Four patients required intubation with mechanical ventilation (three during the initial period and one during the recent period; $\mathrm{p}=0.34$ ). Among those patients, three also required extracorporeal membrane oxygenation support (all during the initial period). Furthermore, a total of six stents were implanted in five patients in the setting of pulmonary artery dissection.

\section{Predictive variables for lung injury}

A comparison of baseline clinical characteristics, haemodynamic variables and PAH therapy between patients with or without lung injury is shown in table 4. Patients who experienced lung injury had significantly higher baseline mPAP and PVR and poorer exercise capacity (6MWD). Factors associated with the occurrence of lung injury after BPA were analysed and are depicted in table 5. Per-patient multivariate analysis revealed that baseline mPAP (OR 1.08, 95\% CI 1.039-1.130; $<<0.001$ ) and the period 
TABLE 2 Clinical and haemodynamic data before and after balloon pulmonary angioplasty

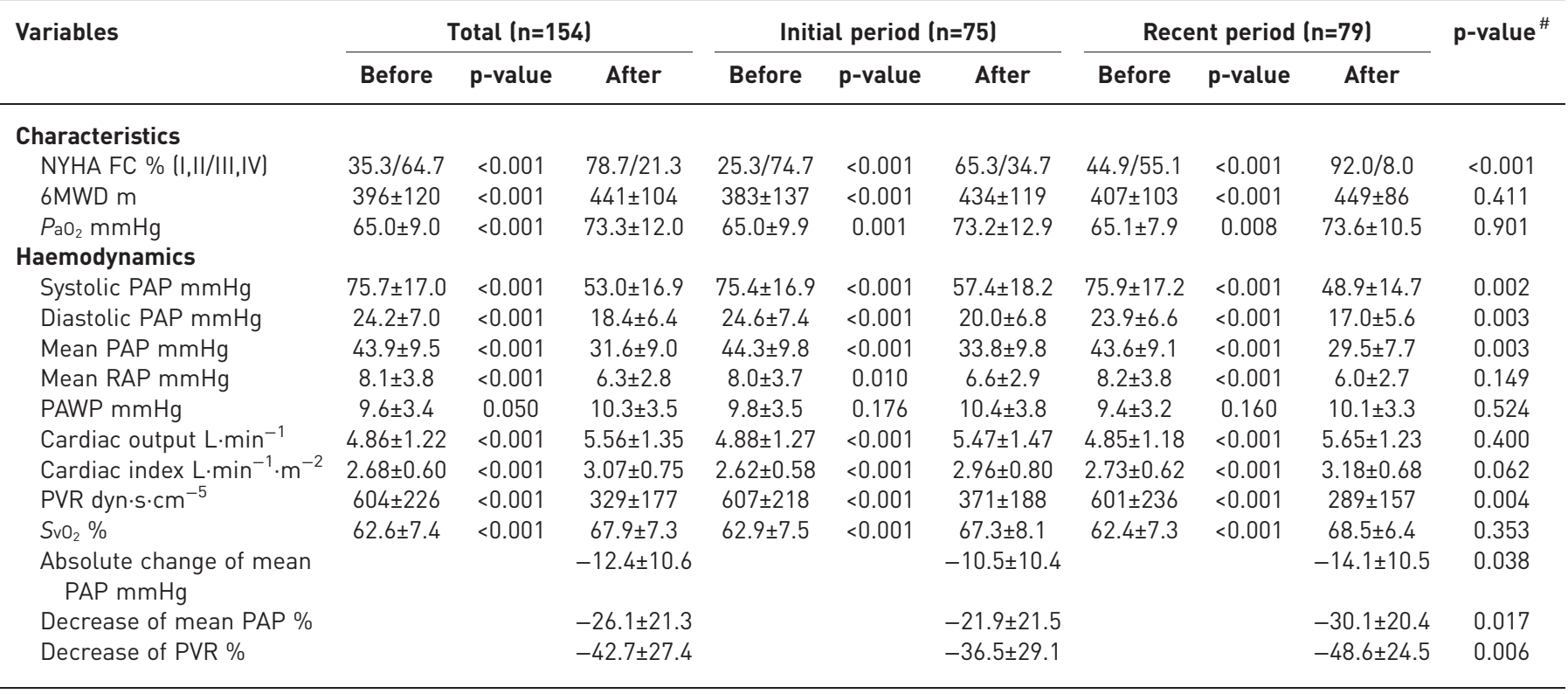

Data are presented as mean \pm SD, unless otherwise stated. NYHA FC: New York Heart Association functional class; 6MWD: 6-min walk distance; $\mathrm{PaO}_{2}$ : arterial partial pressure of oxygen; PAP: pulmonary artery pressure; RAP: right atrial pressure; PAWP: pulmonary artery wedge pressure;

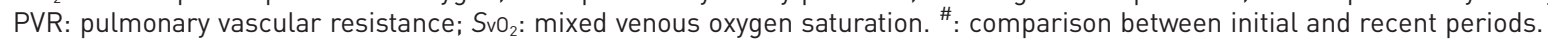

during which BPA procedure was performed (recent versus initial period; OR 0.367, 95\% CI 0.175-0.771; $\mathrm{p}=0.008$ ) were the most significant factors related to lung injury.

\section{Survival}

In total, seven deaths occurred among the 184 patients $(3.8 \%)$. There were four peri-procedural deaths (within 30 days after BPA; 2.2\%) which were related to severe lung injury. Three additional deaths occurred during the follow-up period. Two patients died from right heart failure at 15 and 22 months and one from cancer at 4 months after the first BPA.

Overall survival at 1 and 3 years was 97.3\% (95\% CI 94.9-99.6\%) and 95.1\% (95\% CI 91.4-98.9\%), respectively (figure 2).

\section{Discussion}

In the present study, we report a large series of patients with inoperable CTEPH treated by BPA at the French Reference Centre for Pulmonary Hypertension. In this high volume centre, the programme of PEA for CTEPH was launched in 1996 and, to date, nearly 2000 patients have undergone surgery [30]. Following the

TABLE 3 Complications per session

\begin{tabular}{lcccc} 
Event & Total $(\mathbf{n}=\mathbf{1 0 0 6})$ & Initial period ( $\mathbf{n}=\mathbf{4 4 4 )}$ & Recent period (n=562) & $\mathbf{p}^{\text {-value }}{ }^{\#}$ \\
\hline Overall complications & $113(11.2)$ & $70(15.8)$ & $43(7.7)$ & $<0.001$ \\
Lung injury & $92(9.1)$ & $59(13.3)$ & $33(5.9)$ & $<0.001$ \\
$\quad$ Mild/moderate & $36(3.6)$ & $13(2.9)$ & $23(4.1)$ & 0.394 \\
$\quad$ Severe & $56(5.6)$ & $46(10.4)$ & $10(1.8)$ & $<0.001$ \\
Haemoptysis & $71(7.1)$ & $36(8.1)$ & $35(6.2)$ & 0.266 \\
Pulmonary artery perforation & $28(2.8)$ & $16(3.6)$ & $12(2.1)$ & 0.179 \\
Pulmonary artery dissection & $19(1.9)$ & $9(2.0)$ & $10(1.8)$ & 0.774 \\
NPPV & $49(4.9)$ & $40(9.0)$ & $9(1.6)$ & $<0.001$ \\
Intubation & $4(0.4)$ & $3(0.7)$ & $1(0.2)$ & 0.327 \\
ECMO support & $3(0.3)$ & $3(0.7)$ & $0(0)$ & 0.086 \\
Renal dysfunction & $2(0.2)$ & $2(0.5)$ & $0(0.0)$ & 0.195 \\
\hline
\end{tabular}

Data are presented as $\mathrm{n}(\%)$, unless otherwise stated. NPPV: noninvasive positive pressure ventilation; ECMO: extracorporeal membrane oxygenation. ${ }^{\#}$ : comparison between initial and recent periods. 
TABLE 4 Comparison of characteristics and haemodynamic data between patients with and without lung injury after balloon pulmonary angioplasty

\begin{tabular}{|c|c|c|c|}
\hline Variables & $\begin{array}{l}\text { With lung injury } \\
\qquad(n=63)\end{array}$ & $\begin{array}{l}\text { Without lung injury } \\
\qquad(n=121]\end{array}$ & p-value \\
\hline \multicolumn{4}{|l|}{ Baseline characteristics } \\
\hline Age years & $62.3 \pm 14.8$ & $64.0 \pm 13.3$ & 0.422 \\
\hline Male & $34(54.0)$ & $60(49.6)$ & 0.573 \\
\hline NYHA FC \% (I,II/III,IV) & $28.6 / 71.4$ & $40.0 / 60.0$ & 0.126 \\
\hline 6MWD m & $359 \pm 131$ & $417 \pm 106$ & 0.003 \\
\hline \multicolumn{4}{|l|}{ Baseline haemodynamics } \\
\hline Mean RAP mmHg & $8.7 \pm 3.9$ & $8.1 \pm 4.0$ & 0.415 \\
\hline Mean PAP mmHg & $48.6 \pm 9.7$ & $41.7 \pm 9.0$ & $<0.001$ \\
\hline Cardiac index $\mathrm{L} \cdot \mathrm{min}^{-1} \cdot \mathrm{m}^{-2}$ & $2.55 \pm 0.48$ & $2.79 \pm 0.70$ & 0.014 \\
\hline PVR dyn's.cm $\mathrm{cm}^{-5}$ & $727 \pm 261$ & $549 \pm 231$ & $<0.001$ \\
\hline \multicolumn{4}{|l|}{ PAH therapy at baseline } \\
\hline $\begin{array}{l}\text { No treatment/monotherapy/combination } \\
\text { therapy } \%\end{array}$ & $32 / 25 / 43$ & $41 / 27 / 32$ & 0.274 \\
\hline
\end{tabular}

publication of recent studies of refined BPA and multiple contacts with experienced BPA centres in Japan during 2013, we started a BPA programme for CTEPH in February 2014. For all patients, eligibility for BPA was decided on the basis of a consensus among the CTEPH team during a multidisciplinary meeting.

Our results confirm that BPA is an effective treatment for patients with inoperable CTEPH. Overall, we observed a statistically significant improvement in NYHA functional class, $6 \mathrm{MWD}, \mathrm{PaO}_{2}$ and haemodynamics. Complications related to BPA were observed in $11.2 \%$ of all sessions and $46 \%$ of all patients. As reported in previous studies, lung injury was the most frequent complication $(9.1 \%$ of all sessions and $34 \%$ of patients) and $20.7 \%$ of patients required noninvasive or invasive mechanical ventilation. Four patients $(2.2 \%)$ died within 30 days after BPA (peri-procedural period) and three additional patients died during the follow-up, resulting in a mortality rate of $3.8 \%$.

The average improvement in pulmonary haemodynamics was less pronounced in our study than in the recently reported Japanese registry, with a $43 \%$ and $58 \%$ decrease in PVR, respectively [25]. This difference could be attributed to greater experience with BPA in Japan. However, it cannot be excluded that other factors may also contribute, including differences between French and Japanese patients. Indeed, the Japanese registry included a majority of female patients $(80 \%)$, whereas there was no sex difference in our

TABLE 5 Univariate and multivariate logistic regression analysis of predictive variables of lung injury

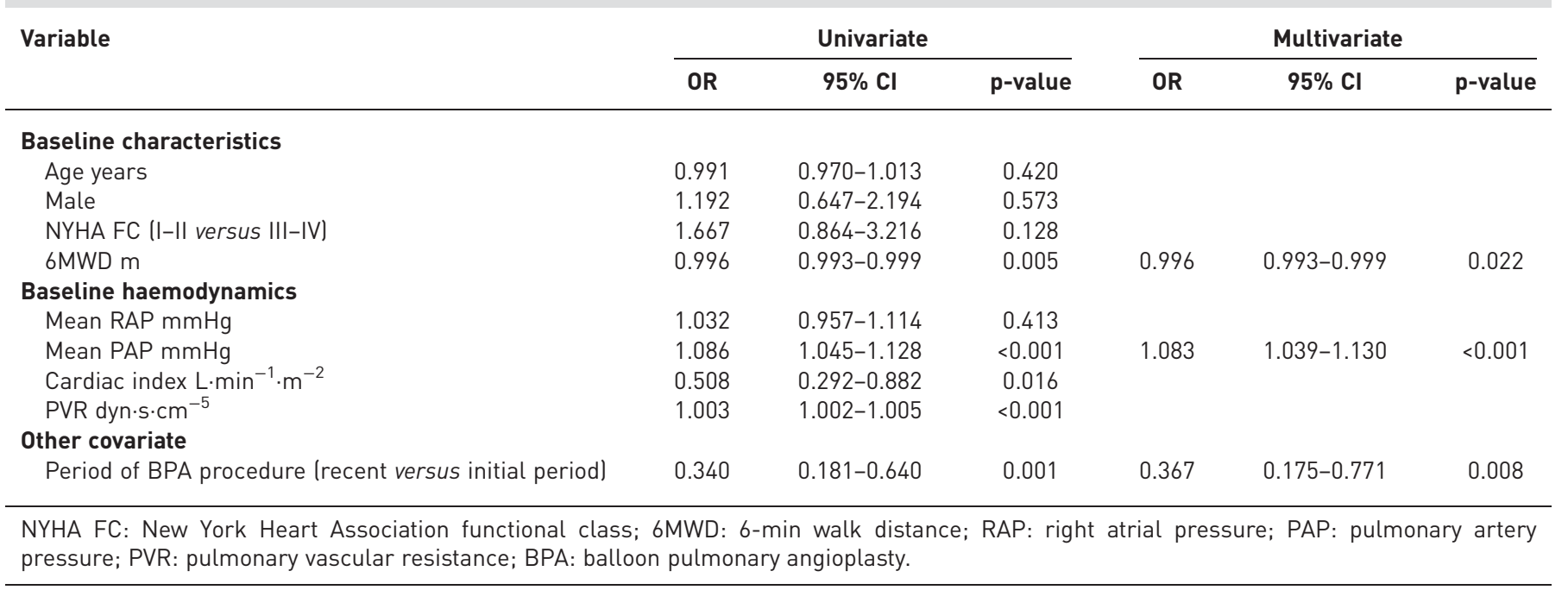


FIGURE 2 Survival from first balloon pulmonary angioplasty session ( $n=184)$.

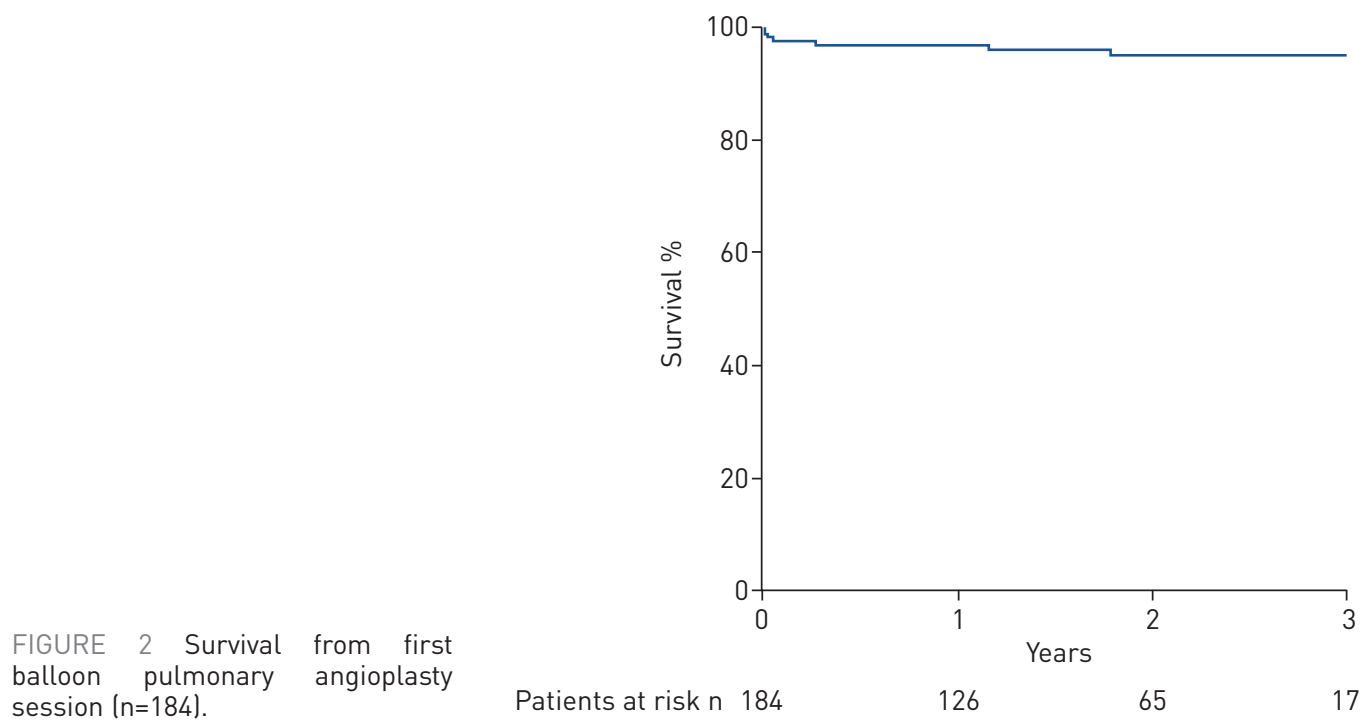

cohort of patients. Furthermore, previous episodes of venous thromboembolism were less frequently found in Japanese patients as compared with our cohort of patients $(34.7 \%$ and $73 \%$, respectively). Finally, fewer Japanese patients were also diagnosed with associated medical conditions increasing the risk of CTEPH. Interestingly, the magnitude of haemodynamic improvement observed in our study was correlated with treatment period. Indeed, the decrease in PVR has improved over time and was significantly greater during the recent period (decrease by $48 \%$ ) than during the initial period (decrease by $37 \%$ ), which is very encouraging. This improvement is mainly due to the experience acquired over time. Thus, over the years, we increased the total number of treated segments in each patient (14.0 during the initial period versus 16 during the recent period) without increasing significantly the number of sessions per patient (5.2 during the initial period versus 5.7 during the recent period). The number of procedures per patient was, however, slightly higher in our study (median 5 procedures) than in the Japanese registry (median 4) [25].

As mentioned previously, complications related to BPA were not rare since they occurred in 113 of 1006 sessions (11.2\% of all sessions and $46 \%$ of all patients). The observed rate of lung injury was lower in our series compared to the Japanese registry (9.1\% versus $17.8 \%$ of all sessions) [25]. Interestingly, the rate of complications recorded in our patient population was also correlated to the treatment period. The frequency of lung injury was reduced from $13.3 \%$ of sessions in the initial period to $5.9 \%$ in the recent period, respectively. Per-patient multivariate analysis evaluating variables associated with lung injury showed that baseline MPAP and the period of BPA procedure (recent versus initial period) were the most significant factors related to lung injury. Thus, patients with a high $\mathrm{MPAP}$ at baseline were at increased risk of developing lung injury after BPA. By contrast, those undergoing BPA in the recent period were at lower risk of lung injury. This significant decrease in lung injury during the recent period is due to the use of a refined BPA strategy developed over time to improve the safety of the procedure. In a recent study, EJIRI et al. [29] showed that BPA-related vascular injury was the main cause and the strongest predictor of lung injury after BPA. In this study, the authors also found that high mPAP before BPA and BPA-related vascular injury were independent risk factors for severe lung injury requiring mechanical ventilation. Insofar as the leading cause of complications is distal vascular injury caused by the tip of the wire, we improved the wiring approach by using the knuckle wire technique to reduce the risk of vascular injury. We also used undersized balloons during the first sessions to prevent balloon injury and, once haemodynamics was improved, we treated target lesions with adequate diameter balloons. Furthermore, since June 2015, we have been using a pressure-wire-guided technique during the first sessions to monitor distal perfusion pressure in patients with crossable lesions and baseline mPAP $\geqslant 45 \mathrm{mmHg}$. This technique allows a measure of the mPAP proximal and distal to the target lesions, and two studies from Japan have shown that the use of pressure-wire-guided technique may reduce the risk of lung injury [20,31].

Other factors might also contribute, including a better approach of target lesions. In 2016, KAWAKAMI et al. [32] proposed an angiographic classification of CTEPH lesions and assessed the rate of BPA-related complications according to the type of lesions. Five types of lesions were described as follows: ring-like stenosis lesion, web lesion, subtotal lesion, total occlusion lesion and tortuous lesion. In this study, the authors showed that complication rate was highly dependent on the lesion characteristics. The vascular complication rate was lower in ring-like stenosis and web lesions and was higher with tortuous lesions. 
The type of lesion was not recorded in our study because the initial period was completed before the lesion distribution was classified and published by KAWAKAMI et al. [32]. However, our group targeted, as a priority, ring-like stenosis, web and subtotal lesions, and this careful approach has not changed between the initial and recent periods. Furthermore, in the study published by EJIRI et al. [29] in 2018, the authors did not find any correlation between the angiographic type of treated lesion and the occurrence of lung injury. Therefore, all these findings suggest that the operators' experience, together with the refinement of BPA technique, both played a key role in decreasing the risk of BPA-related complications.

The observed mortality of $3.8 \%$ in our series is similar to the $3.9 \%$ reported by the Japanese registry [25]. In these two studies, the majority of deaths were observed during the peri-procedural period. In our series, peri-procedural mortality rate was lower in the recent period than in the initial period (1\% versus $3.5 \%$ ) and no peri-procedural death occurred over the final year of the study. Several factors may have contributed to this improvement in survival, including better selection of candidates for BPA, careful wiring approach, use of pressure-wire-guided technique and undersized balloons during the first sessions, and better use of PAH-targeted therapy before BPA.

Our study has several limitations. Firstly, it is a single centre study. Secondly, there is no control group receiving only medical therapy during the same period. Thirdly, as previously reported in other studies, a high proportion of our patients (62\%) were receiving at least one PAH-targeted therapy for at least 3 months before the first BPA session [16-20, 22-26]. One could assume that the use of medical therapy before BPA may have an impact on post-procedural outcome. Our study, unfortunately, does not allow any answers to be given to this issue. Indeed, our patients were not randomly assigned to receive either PAH-targeted drugs or no PAH-targeted drugs before BPA. Lastly, we do not have any results of long-term follow-up of more than 3 years; therefore, there might be cases of $\mathrm{PH}$ worsening after this period.

\section{Conclusions}

We report here the largest single centre experience of BPA outside Japan, which is the pioneering country regarding this interventional technique. Our results confirm that a refined BPA significantly improves short-term symptoms, oxygenation, exercise capacity and haemodynamics in inoperable CTEPH patients. We observed that lung injury was the main peri-procedural complication and we demonstrated that high mPAP before BPA was a risk factor for lung injury. In our series, safety and efficacy have improved over time, underscoring the unavoidable learning curve period for this complex procedure even in a high volume and experienced CTEPH centre with a multidisciplinary approach. However, although peri-procedural mortality is acceptable, the rate of complications remains high and safety of the procedure can be further improved. In addition, long-term effects of BPA need to be properly evaluated in a prospective, multicentre study.

Conflict of interest: P. Brenot has nothing to disclose. X. Jais reports grants and personal fees from Actelion, GSK, Bayer and MSD, outside the submitted work. Y. Taniguchi has nothing to disclose. C. Garcia Alonso has nothing to disclose. B. Gerardin has nothing to disclose. S. Mussot has nothing to disclose. O. Mercier has nothing to disclose. D. Fabre has nothing to disclose. F. Parent has nothing to disclose. M. Jevnikar has nothing to disclose. D. Montani reports grants and personal fees from Actelion and Bayer, personal fees from GSK, Pfizer, BMS and MSD, outside the submitted work. L. Savale reports grants, personal fees and non-financial support from Actelion and GSK, personal fees and non-financial support from MSD, outside the submitted work. O. Sitbon reports grants, personal fees and non-financial support from Actelion Pharmaceuticals and GlaxoSmithKline, grants and personal fees from Bayer HealthCare, grants and non-financial support from Merck, personal fees from Arena Pharmaceuticals, outside the submitted work. E. Fadel has nothing to disclose. M. Humbert reports grants and personal fees from Actelion, Bayer, GSK and from MSD, personal fees from Johnson \& Johnson and United Therapeutics, outside the submitted work. G. Simonneau reports grants, personal fees and non-financial support from Actelion Pharmaceuticals, Bayer Healthcare, Merck and GlaxoSmithKline, outside the submitted work.

\section{References}

1 Kim NH. Group 4 Pulmonary Hypertension: Chronic Thromboembolic Pulmonary Hypertension: Epidemiology, Pathophysiology, and Treatment. Cardiol Clin 2016; 34: 435-441.

2 Simonneau G, Torbicki A, Dorfmuller P, et al. The pathophysiology of chronic thromboembolic pulmonary hypertension. Eur Respir Rev 2017; 26: 160112.

3 Sharma S, Lang IM. Current understanding of the pathophysiology of chronic thromboembolic pulmonary hypertension. Thromb Res 2018; 164: 136-144.

4 Fedullo P, Kerr KM, Kim NH, et al. Chronic thromboembolic pulmonary hypertension. Am J Respir Crit Care Med 2011; 183: 1605-1613.

5 Hoeper MM, Madani MM, Nakanishi N, et al. Chronic thromboembolic pulmonary hypertension. Lancet Respir Med 2014; 2: 573-582.

6 Corsico AG, D’Armini AM, Cerveri I, et al. Long-term outcome after pulmonary endarterectomy. Am J Respir Crit Care Med 2008; 178: 419-424.

7 Condliffe R, Kiely DG, Gibbs JS, et al. Improved outcomes in medically and surgically treated chronic thromboembolic pulmonary hypertension. Am J Respir Crit Care Med 2008; 177: 1122-1127. 
8 Madani MM, Auger WR, Pretorius V, et al. Pulmonary endarterectomy: recent changes in a single institution's experience of more than 2700 patients. Ann Thorac Surg 2012; 94: 97-103.

9 Cannon JE, Su L, Kiely DG, et al. Dynamic risk stratification of patient long-term outcome after pulmonary endarterectomy: results from the United Kingdom national cohort. Circulation 2016; 133: 1761-1771.

10 Pepke-Zaba J, Delcroix M, Lang I, et al. Chronic thromboembolic pulmonary hypertension (CTEPH): results from an international prospective registry. Circulation 2011; 124: 1973-1981.

11 Delcroix M, Lang I, Pepke-Zaba J, et al. Long-term outcome of patients with chronic thromboembolic pulmonary hypertension: results from an international prospective registry. Circulation 2016; 133: 859-871.

12 Galie N, Humbert M, Vachiery JL, et al. 2015 ESC/ERS Guidelines for the diagnosis and treatment of pulmonary hypertension: The Joint Task Force for the Diagnosis and Treatment of Pulmonary Hypertension of the European Society of Cardiology (ESC) and the European Respiratory Society (ERS). Eur Respir J 2015; 46: 903-975.

13 Ogawa A, Matsubara H. After the dawn: balloon pulmonary angioplasty for patients with chronic thromboembolic pulmonary hypertension. Circ J 2018; 82: 1222-1230.

14 Hosking MC, Thomaidis C, Hamilton R, et al. Clinical impact of balloon angioplasty for branch pulmonary arterial stenosis. Am J Cardiol 1992; 69: 1467-1470.

15 Feinstein JA, Goldhaber SZ, Lock JE, et al. Balloon pulmonary angioplasty for treatment of chronic thromboembolic pulmonary hypertension. Circulation 2001; 103: 10-13.

16 Mizoguchi H, Ogawa A, Munemasa M, et al. Refined balloon pulmonary angioplasty for inoperable patients with chronic thromboembolic pulmonary hypertension. Circ Cardiovasc Interv 2012; 5: 748-755.

17 Sugimura K, Fukumoto Y, Satoh K, et al. Percutaneous transluminal pulmonary angioplasty markedly improves pulmonary hemodynamics and long-term prognosis in patients with chronic thromboembolic pulmonary hypertension. Circ J 2012; 76: 485-488.

18 Taniguchi Y, Miyagawa K, Nakayama K, et al. Balloon pulmonary angioplasty: an additional treatment option to improve the prognosis of patients with chronic thromboembolic pulmonary hypertension. EuroIntervention 2014; 10: $518-525$

19 Yanagisawa R, Kataoka M, Inami T, et al. Safety and efficacy of percutaneous transluminal pulmonary angioplasty in elderly patients. Int J Cardiol 2014; 175: 285-289.

20 Inami T, Kataoka M, Shimura N, et al. Pressure-wire-guided percutaneous transluminal pulmonary angioplasty: a breakthrough in catheter-interventional therapy for chronic thromboembolic pulmonary hypertension. JACC Cardiovasc Interv 2014; 7: 1297-1306.

21 Inami $\mathrm{T}$, Kataoka $\mathrm{M}$, Shimura $\mathrm{N}$, et al. Incidence, avoidance, and management of pulmonary artery injuries in percutaneous transluminal pulmonary angioplasty. Int J Cardiol 2015; 201: 35-37.

22 Inami $\mathrm{T}$, Kataoka $\mathrm{M}$, Yanagisawa $\mathrm{R}$, et al. Long-term outcomes after percutaneous transluminal pulmonary angioplasty for chronic thromboembolic pulmonary hypertension. Circulation 2016; 134: 2030-2032.

23 Ogo T, Fukuda T, Tsuji A, et al. Efficacy and safety of balloon pulmonary angioplasty for chronic thromboembolic pulmonary hypertension guided by cone-beam computed tomography and electrocardiogram-gated area detector computed tomography. Eur J Radiol 2017; 89: 270-276.

24 Aoki T, Sugimura K, Tatebe S, et al. Comprehensive evaluation of the effectiveness and safety of balloon pulmonary angioplasty for inoperable chronic thrombo-embolic pulmonary hypertension: long-term effects and procedure-related complications. Eur Heart J 2017; 38: 3152-3159.

25 Ogawa A, Satoh T, Fukuda T, et al. Balloon pulmonary angioplasty for chronic thromboembolic pulmonary hypertension: results of a multicenter registry. Circ Cardiovasc Qual Outcomes 2017; 10: e004029.

26 Olsson KM, Wiedenroth CB, Kamp JC, et al. Balloon pulmonary angioplasty for inoperable patients with chronic thromboembolic pulmonary hypertension: the initial German experience. Eur Respir J 2017; 49: 1602409.

27 Kim NH, Delcroix M, Jais X, et al. Chronic thromboembolic pulmonary hypertension. Eur Respir J 2019; 53: 1801915.

28 Matsubara H, Ogawa A. A long way to go after the initial experience with balloon pulmonary angioplasty. Eur Respir J 2017; 49: 1700718.

29 Ejiri K, Ogawa A, Fujii S, et al. Vascular injury is a major cause of lung injury after balloon pulmonary angioplasty in patients with chronic thromboembolic pulmonary hypertension. Circ Cardiovasc Interv 2018; 11: e005884.

30 Dartevelle P, Fadel E, Mussot S, et al. Chronic thromboembolic pulmonary hypertension. Eur Respir J 2004; 23 : 637-648.

31 Kinutani H, Shinke T, Nakayama K, et al. High perfusion pressure as a predictor of reperfusion pulmonary injury after balloon pulmonary angioplasty for chronic thromboembolic pulmonary hypertension. Int J Cardiol Heart Vasc 2015; 11: 1-6.

32 Kawakami T, Ogawa A, Miyaji K, et al. Novel angiographic classification of each vascular lesion in chronic thromboembolic pulmonary hypertension based on selective angiogram and results of balloon pulmonary angioplasty. Circ Cardiovasc Interv 2016; 9: e003318. 